\title{
Comprensión individual y diádica de tablas de frecuencias en alumnos de escolaridad primaria ${ }^{1}$
}

\author{
Mariano Castellaro ${ }^{2}$ \\ Instituto Rosario de Investigaciones en Ciencias de la Educación / \\ Universidad Nacional de Rosario (Argentina) \\ Néstor Roselli ${ }^{3}$ \\ Centro de Investigaciones en Psicología, Pontificia Universidad Católica Argentina / \\ Consejo Nacional de Investigaciones Científicas y Técnicas (Argentina)
}

Recibido: 21/02/2019Ａceptado: 13/12/2019

\begin{abstract}
Resumen
Objetivo. Analizar la integración entre comprensión individual y diádica de una tabla de frecuencias en estudiantes de primaria, cuyo foco estuvo en la calidad y cualidad del producto cognitivo. Método. Participaron 90 estudiantes de $6^{\circ}$ y $7^{\circ}$ grado (edad: $M=12.3 ; D E=0.5$ ), seleccionados no probabilísticamente. Primero, los sujetos resolvieron de forma individual la tarea de comprensión de una tabla de doble entrada. Luego fueron agrupados en díadas que resolvieron la tarea colaborativamente. Se utilizó un cuestionario para evaluar niveles de complejidad de dicha comprensión y centrar el análisis en: (a) la comparación de desempeños individual y diádico generales, (b) las modalidades de integración entre producción individual y diádica, y (c) las modalidades según complejidad del ítem y composición sociocognitiva diádica. Resultados. Se encontró: (a) superioridad del desempeño diádico sobre el individual, (b) se identificaron diferentes modalidades de integración entre comprensión individual y diádica, y (c) la influencia del nivel de complejidad del ítem y la composición sociocognitiva sobre modalidades específicas de respuesta construidas por la díada. Conclusión. Se corrobora la hipótesis de la superioridad de las actuaciones interindividuales sobre las intraindividuales, aunque con variaciones generadas por las variables mediadoras. Los hallazgos se discutieron según la diferenciación conceptual entre producto y proceso.
\end{abstract}

Palabras clave. Interacción social, comprensión, tablas, representación del conocimiento.

\footnotetext{
El presente estudio es parte del proyecto "Factores psicosociales de la colaboración socio-cognitiva entre pares. Hacia un enfoque situado de la cognición colectiva", financiado por el Consejo Nacional de Investigaciones Científicas y Técnicas (CONICET) y radicado en el Instituto Rosario de Investigaciones en Ciencias de la Educación (IRICE), de Argentina.

2 Doctor en Psicología. Información de correspondencia: 27 de febrero 210 bis (Ocampo y Esmeralda), Rosario, Argentina (C.P. 2000). Tel.: +54-3414821769 (Int. 210). Correo de correspondencia: castellaro@irice-conicet.gov.ar

3 Doctor en Psicología.
} 


\title{
Individual and Collaborative Comprehension of Frequency Tables in Primary School Students
}

\begin{abstract}
Objective. To analyze the integration between individual and dyadic comprehension of frequency tables in primary school students. The focus was on the quality and modality of cognitive product (final solution). Method. The participants were 90 sixth and seventh grade students (age: $M=12.3 ; S D=0.5$; non-probabilistic sample). First, the subjects individually solved a comprehension task from a double-entry table. Then they were grouped into dyads which solved the task collaboratively. A questionnaire was used to evaluate levels of task comprehension and analyze: (a) comparison of individual and dyadic performances; (b) modalities of integration between individual and dyadic production; (c) analysis of modalities according to item and dyadic socio-cognitive composition. Results. Data showed: (a) the superiority of the collaborative performance compared to the individual performance; (b) different modalities of integration between both individual and dyadic comprehension; and (c) the level of complexity of the item and the socio-cognitive composition of the dyad influenced specific modalities. Conclusion. The hypothesis of the superiority of inter-individual actions over intra-individual actions was corroborated, although with variations associated with the mediating variables. The findings were discussed with a differentiation between product and process.
\end{abstract}

Keywords. Social interaction, comprehension, tables, knowledge representation.

\section{Compreensão individual e diádica de tabelas de frequências em alunos de escolaridade primária}

\section{Resumo}

Escopo. Analisar a integração entre compreensão individual e diádica de uma tabela de frequência em estudantes de primária, com o foco na qualidade do produto cognitivo. Metodologia. Participaram 90 estudantes de $6^{\circ}$ e $7^{\circ}$ grau (idade $M=12.3 ; S D=0.5$ ), selecionados de jeito não probabilístico. Primeiro, os sujeitos resolveram de forma individual a tarefa de compreensão de uma tabela de dupla entrada. Depois foram agrupados em díadas que resolveram a tarefa de modo colaborativo. Foi utilizado um questionário para avaliar níveis de complexidade na compreensão. Análises efetuados: (a) comparação de desempenhos individual e diádico gerais, (b) modalidades de integração entre produção individual y diádica, e (c) modalidades segundo a complexidade do item e composição sociocognitiva diádica. Resultados. Se encontrou (a) superioridade do desempenho diádico sobre o individual, (b) foram identificadas diferentes modalidades de integração entre compreensão individual e diádica, e (c) o nível de complexidade do item e a composição sociocognitiva influíram sobre modalidades específicas de resposta construída pela díada. Conclusão. Foi corroborada a hipóteses da superioridade das atuações inter-individuais sobre as intra-individuais, mesmo que com variações geradas pelas variáveis mediadoras. As descobertas foram discutidas segundo a diferenciação conceitual entre produto e processo.

Palavras-chave. Interação social, compreensão, tabelas, representação do conhecimento

\section{Introducción}

La interacción entre pares constituye un objeto de estudio recurrente en la psicología del desarrollo y en la psicología de la educación. Dicho interés se fundamenta en la estrecha relación que se registra entre este tipo de interacción y el cambio cognitivo (Psaltis y Zapiti, 2014). En general, las investigaciones se han enfocado en analizar las características de la interacción sociocognitiva entre pares y las consecuencias cognitivas individuales derivadas de esta (Castellaro, 2017; Castellaro y Roselli, 2015). 
Sin embargo, hay otro aspecto del fenómeno que, si bien tiene directa relación con los dos anteriores, ha sido menos atendido: la comparación entre la perspectiva cognitiva individual sobre la tarea solución propuesta previa a la interacción- y la perspectiva coconstruida posteriormente a partir del trabajo diádico. Es decir, es necesario entender ¿cómo se traslada la posición individual de cada uno de los sujetos sobre el problema, a la posición colectiva construida luego por ambos? y ¿cómo se produce esta transición e integración individuodíada en términos de calidad (correcta o incorrecta) y cualidad (tipo o modalidad)?

A su vez, la comparación entre producto cognitivo individual y producto cognitivo diádico conlleva otra pregunta: ¿Dos mentes logran mejores producciones que una sola? Esta cuestión concierne a la superioridad de la resolución, desempeño o performance colectiva de una tarea respecto a la resolución individual, lo cual constituyó uno de los interrogantes iniciales del socioconstructivismo europeo (Gilly, 1992; Peralta y Castellaro, 2018). La hipótesis de base sostiene que la resolución de una tarea en condición colectiva, por ejemplo, díada, implica un producto cognitivo superior al logrado en condición individual (Peralta, Roselli y Borgobello, 2012; Roselli, 2016). Aunque esta pregunta tiene un sentido principalmente teórico, al mismo tiempo posee implicancias prácticas vinculadas con el aprendizaje escolar, en tanto recupera el debate entre posiciones individualistas e interaccionistas sobre la construcción del conocimiento.

Debe aclararse que la construcción sociocognitiva, que se refiere tanto al propio proceso interactivo como al producto construido, puede entenderse de dos maneras: como negociación de posiciones individuales que tratan de llegar a un acuerdo, y como colaboración, entendida como construcción colectiva auténtica (MejíaArauz, Rogoff, Dayton y Henne-Ochoa, 2018). La primera define el fenómeno de la coconstrucción colectiva como el resultado directo de la adición de comportamientos individuales o de la coordinación entre puntos de vista. Así, este logro implica una tensión o conflicto entre las propias posiciones epistémico-sociales de los sujetos que interactúan, cuya resolución exige un consenso basado en el intercambio de argumentos.

El modelo de colaboración, por su parte, enfatiza el carácter de complementariedad interpersonal de la coconstrucción colectiva. En otras palabras, la construcción de un campo de significación compartida sobre la tarea constituye un emergente intersubjetivo natural, irreductible a los posicionamientos puramente individuales. A pesar de esta diferenciación, y dado que en el presente trabajo no se analiza el proceso de construcción sociocognitiva, sino la oposición entre producto sociocognitivo individual y diádico, no hay elementos para diferenciar entre negociación y colaboración intersubjetiva auténtica. De tal manera que, para evitar una confusión entre ambas conceptualizaciones, se apelará directamente al término genérico diádico para analizar el producto (socio)cognitivo logrado a partir de una instancia interactiva, en contraposición con un producto cognitivo logrado en una condición de individualidad.

En este artículo, la comparación entre producto cognitivo individual y el producto cognitivo diádico es efectuada en un tipo específico de tarea, la comprensión de tablas de frecuencias. Esta decisión tiene su fundamentación no solo en el hecho de que es una tarea muy poco considerada en la literatura sobre interacción entre pares, sino, principalmente, porque es parte del conjunto de sistemas de representación externa que, junto al lenguaje, constituyen las bases centrales aportadas por el sistema cultural para la construcción del conocimiento (Gabucio, Martí, Enfedaque, Gilabert y Konstantinidou, 2010). En otras palabras, la elección de esta actividad se inscribe en un propósito teórico novedoso, el cual establece conexiones conceptuales entre las líneas de investigación sobre colaboración entre pares (Psaltis, Duveen y Perret-Clermont, 2009), por un lado, y las líneas de investigación sobre sistemas externos de representación (Pérez, Martí y Pozo, 2010), por el otro. Así, el trabajo es parte de una intención mayor, de largo plazo, que consiste en explorar diferentes procesos (interpretación, construcción y utilización) ligados a diversos sistemas externos de representación, siempre en el marco de interacciones entre pares. Hasta el momento, la mayor parte de los estudios sobre comprensión, construcción o uso de sistemas externos de representación estuvieron enfocados en resoluciones individuales (García-Mila, Martí, Gilabert y Castells, 2014; Pérez-Echeverría, Postigo y Marín, 2010) y en la interacción adultoniño (Peralta y Salsa, 2011; Peralta, Salsa, Maita y Mareovich, 2013; Salsa y Peralta, 2009). 
El enfoque interaccionista del conocimiento asume que este se produce necesariamente en torno a una mediación representacional externa, soporte objetivo o material, el cual puede involucrar diferentes sistemas. Estos pueden implicar tanto el lenguaje escrito como otros sistemas externos permanentes, cada uno definido por características particulares y niveles propios de complejidad, por ejemplo, la notación numérica (Martí, Scheuer, Cavalcante, Trench y Brizuela, 2016; Rodríguez, Martí y Salsa, 2018), los gráficos (Salsa y Gariboldi, 2017; Salsa y Vivaldi, 2017) y los mapas (Maita, Mareovich y Peralta, 2014), entre otros. Dentro de esta variedad, las tablas de frecuencias aluden a un sistema específico, cuya representación externa u objetiva del conocimiento se hace a través de un formato bidimensional que contiene cantidades numéricas (Gabucio et al., 2010; Martí, GarcíaMila, Gabucio y Konstantinidou, 2011). En la educación formal, los procesos de apropiación de este tipo de sistemas comienzan hacia finales del ciclo primario. Desde el punto de vista cognitivo, las tablas de frecuencias de doble entrada, como la del presente estudio, permiten diferenciar los grados de comprensión y modalidades de lectura. Esto las hace diferentes de las clásicas pruebas de resolución de problemas u otras de algoritmo único.

El objetivo de este estudio fue analizar la integración entre comprensión individual y comprensión diádica de una tabla de frecuencias. El interés estuvo centrado en la calidad (correcto/ incorrecto) y la cualidad (tipo o clase de respuesta) del producto cognitivo logrado en ambas condiciones (Roselli, 2016). Además, la comparación y la integración entre comprensión individual y diádica de tablas de frecuencias también fueron analizadas en función de dos variables intervinientes, habitualmente consideradas dentro de la literatura: (a) el grado de dificultad de los ítems del problema y (b) el nivel de competencia previa de los integrantes de la díada. En cuanto al primero, es probable que el grado de dificultad de los ítems genere variaciones significativas, tal como lo sugieren diversos antecedentes referidos a otras tareas (Arterberry, Cain y Chopko, 2007; Liskala, Vauras, Lehtinen y Salonen, 2011). Se diferenciaron cuatro niveles de dificultad de los ítems, en función del menor o mayor grado de complejidad exigido en la comprensión de la tabla (Gabucio et al., 2010): (a) describir datos de la tabla, (b) comprender la estructura tabular, (c) inferir datos particulares de la tabla, e (d) inferir aspectos globales de la tabla.

Por otra parte, la composición sociocognitiva de las díadas se refiere a la igualdad o diferencia entre las competencias específicas de los compañeros de trabajo, lo que se traduce como simetría o asimetría cognitiva (Asterhan, Schwarz y CohenEliyahu, 2014; Buchs y Butera, 2009; Castellaro y Roselli 2018a, b; Schmitz y Winskel, 2008). En este sentido, interesa analizar si este aspecto se asocia con formas diferenciales de integración entre comprensión individual y diádica de la tabla.

En síntesis, los objetivos específicos, todos referidos a la tarea de comprensión de la tabla, fueron los siguientes: (a) comparar los desempeños (cantidad de ítems resueltos correctamente) individual y diádico; (b) analizar las modalidades de integración entre las respuestas individuales de ambos compañeros (previo a la interacción) y la respuesta diádica posterior; (c) analizar las modalidades identificadas en el objetivo b, en función del grado de complejidad de los ítems (cuatro niveles mencionados); y (d) analizar las modalidades identificadas en el objetivo b, en función de la composición sociocognitiva de la díada (simétrica y asimétrica).

\section{Método}

\section{Diseño}

El diseño fue cuasiexperimental, el cual incluyó una fase de pretest individual, en la que cada participante resolvió una tarea de comprensión de una tabla de frecuencias. Sobre la base de esa evaluación inicial, se conformaron dos tipos de díada según el nivel de competencia específica de sus integrantes: simétricas y asimétricas (ver Procedimiento). En la segunda fase, de carácter colectivo, cada díada resolvió la misma tarea utilizada en el pretest individual.

\section{Participantes}

Participaron 90 individuos (45 díadas) de $6^{\circ}$ y $7^{\circ}$ grado de escolaridad primaria (edad en años, $M=12.30 ; D E=0.50$ ) de dos escuelas de Rosario (Argentina), seleccionados de manera no probabilística. El perfil sociocultural predominante 
correspondió a sujetos residentes en sectores urbanizados, en una de las ciudades con mayor índice poblacional del país y ubicada en la zona central del mismo. En su gran mayoría, pertenecían a un nivel socioeconómico medio. En cuanto al género, se distribuyeron equitativamente, $49 \%$ mujeres y $51 \%$ varones. Con respecto al curso, el $44 \%$ de los participantes correspondía a $6^{\circ}$ grado y el resto $(56 \%)$ a $7^{\circ}$ grado. Por último, en relación con la composición sociocognitiva de las díadas, 24 (53\%) fueron simétricas y 21 (47\%) asimétricas.

\section{Materiales}

Los sujetos resolvieron una tarea de comprensión de tabla de frecuencias, idéntica a la propuesta por Gabucio et al. (2010), primero de forma individual y luego en díada. Dicha tabla era de doble entrada y resumía distribuciones de frecuencias de un grupo ficticio de alumnos en función de dos variables: peso (columnas) y sexo (filas) (tabla 1 ).

Tabla 1

Tabla utilizada en la tarea de comprensión

\begin{tabular}{lcccc}
\hline & $\begin{array}{c}\text { Menos de } \\
25 \mathrm{~kg} .\end{array}$ & $\begin{array}{c}\text { De } 25 \\
\text { a } 34 \mathrm{~kg} .\end{array}$ & $\begin{array}{c}\text { De } 35 \\
\text { a } 44 \mathrm{~kg} .\end{array}$ & $\begin{array}{c}\text { Más de } \\
44 \mathrm{~kg} .\end{array}$ \\
\hline Niños & 1 & 10 & 12 & 2 \\
Niñas & 0 & 6 & 13 & 6 \\
Totales & 1 & 16 & 25 & 8 \\
\hline
\end{tabular}

Fuente: Gabucio et al. (2010).

A partir de la lectura de la tabla, los sujetos debían responder un cuestionario de 12 preguntas de opción múltiple, cada una con cuatro opciones de respuesta, de las cuales solo una era correcta. Cada uno de los 12 ítems indagaba uno de los cuatro aspectos o niveles de comprensión del sistema representacional, los cuales variaban en cuanto al grado de complejidad, de menor a mayor: descripción de datos de la tabla (ítems 4, 5 y 10), comprensión de la estructura tabular (ítems 1, 2 y 3), inferencia particular de datos de la tabla (ítems 7,8 , 9 y 11) e inferencia global de la tabla (ítems 6 y 12). Este orden de complejidad entre los cuatro aspectos no existía al interior de cada uno, ya que cada ítem tenía un valor de complejidad equivalente. La confiabilidad del instrumento fue determinada como aceptable por Gabucio et al. (2010).

\section{Procedimiento}

Inicialmente, se obtuvieron los consentimientos informados de la institución escolar, de los padres y de los propios participantes. No participaron del estudio quienes no contaban con alguna de estas autorizaciones. Al interior de cada curso, los sujetos realizaron individualmente la tarea de comprensión de la tabla. Sobre esta base se diferenciaron sujetos con dos niveles de comprensión general de la tabla, básico y avanzado; lo cual se determinó a partir de un criterio gradual correlativo, un sujeto era considerado con cierto nivel general de comprensión solo si demostraba respuestas correctas en los ítems correspondientes a los niveles menos complejos. Esto se realizó con el fin de controlar el efecto de respuestas azarosas. En la tabla 2 se describen los criterios correlativos utilizados para determinar el nivel general de comprensión de la tabla.

Tabla 2

Criterios correlativos para evaluar el nivel general de comprensión de la tabla

\begin{tabular}{|c|c|c|c|c|}
\hline \multirow{3}{*}{ Criterios correlativos de comprensión } & \multicolumn{4}{|c|}{ Nivel general de comprensión } \\
\hline & \multicolumn{2}{|c|}{ Básico } & \multicolumn{2}{|c|}{ Avanzado } \\
\hline & Nivel 1 & Nivel 2 & Nivel 3 & Nivel 4 \\
\hline$\leq 3$ ítems correctos de lectura descriptiva & $\mathrm{x}$ & $\mathrm{x}$ & $\mathrm{X}$ & $\mathrm{X}$ \\
\hline 2-3 ítems correctos de comprensión de la estructura tabular & & $x$ & $x$ & $x$ \\
\hline 2-4 ítems correctos de inferencia de datos & & & $x$ & $x$ \\
\hline 1-2 ítems correctos de inferencia global & & & & $x$ \\
\hline
\end{tabular}

Fuente: elaboración propia. 
A partir de esta evaluación, se conformaron las díadas simétricas y asimétricas. Las primeras estuvieron integradas por dos sujetos de competencia básica (nivel 1 o 2); las segundas, por un sujeto de competencia básica (nivel 1 o 2) y otro de competencia avanzada (nivel 3 o 4), entre los cuales existía una diferencia mínima de dos niveles, por ejemplo, un sujeto de nivel 1 con otro sujeto de nivel 3 o 4, o uno de nivel 2 con otro de nivel 4 .

Durante las dos o tres semanas posteriores, se solicitaba a cada díada que resolviera la misma tarea realizada en la fase individual, solo que en esta instancia la consigna era claramente colaborativa: ambos compañeros debían resolver la actividad de manera conjunta, discutiendo acerca de la solución correcta de cada pregunta para lograr una decisión compartida. Para ello no se estableció un tiempo límite. La actividad tuvo lugar en el horario habitual de clase, en un espacio diferente al aula ordinaria de actividades.

\section{Análisis de los datos}

Se definieron cuatro pasos analíticos, correspondientes a los cuatro objetivos específicos planteados, que se describen a continuación:
1. Comparación de los desempeños (ítems resueltos correctamente) individual y diádico (comparación de medias; prueba $t$ de student para muestras relacionadas).

2. Análisis de las modalidades de integración entre respuestas individuales y respuesta diádica de cada díada. Si bien la unidad de análisis fue cada díada, la integración entre las respuestas individuales y la colectiva se analizó en cada uno de los 12 ítems realizados. Para la categorización de las formas de integración individuo-díada, se tomó como referencia inicial tanto la calidad de las respuestas (correcta/incorrecta) como su cualidad (iguales/ diferentes entre sí). De esta forma, en relación con cada ítem, hubo tres tipos de situaciones iniciales: (a) ambos sujetos habían presentado respuestas individuales correctas, (b) un sujeto había presentado una respuesta correcta y el otro, una respuesta incorrecta, (c) ambos sujetos habían presentado respuestas individuales incorrectas. Así, al interior de cada una de estas situaciones iniciales $(\mathrm{a}, \mathrm{b}$ o c), se distinguieron modalidades específicas según la respuesta colectiva construida posteriormente por la díada. En la tabla 3 se presentan todas las modalidades reconocidas.

Tabla 3

Modalidades de integración entre comprensión individual (respuestas de ambos sujetos previas a la interacción) y comprensión diádica posterior

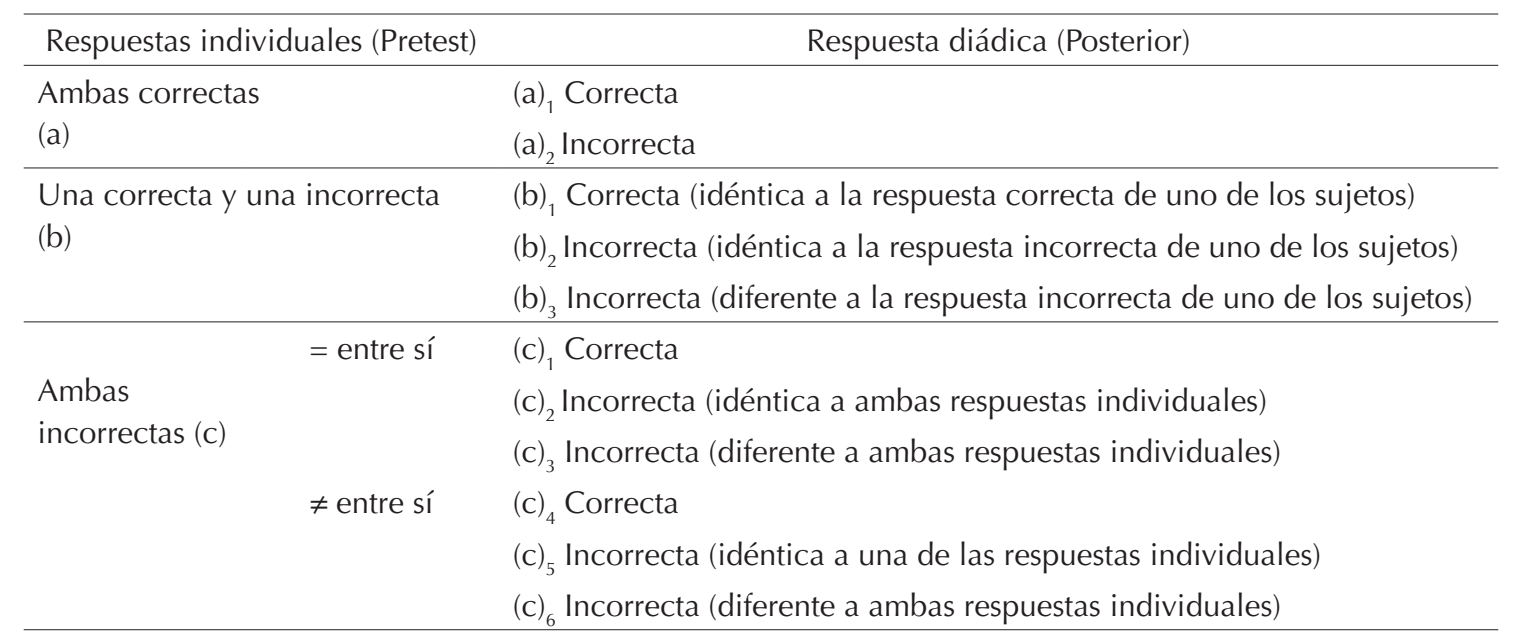

Fuente: elaboración propia.

Con relación a cada díada, se calculó la proporción de ocurrencia (entre 0 y 1 ) de cada modalidad de integración individual-colectiva. La proporción de ocurrencia de cada modalidad fue calculada sobre el total de ítems de su misma clase, o sea, (a), (b) o (c). Por ejemplo, en los ítems en 
cuyo pretest un sujeto había mostrado una respuesta correcta y el compañero una respuesta incorrecta (situación b), se calculó qué proporción de estos, en la fase diádica, mantuvo la respuesta correcta del primero $\left[(b)_{1}\right]$, mantuvo la respuesta incorrecta del otro compañero $\left[(b)_{2}\right]$ o propuso una respuesta incorrecta inédita con respecto al pretest $\left[(b)_{3}\right]$. Entonces, si una díada registraba un total de cinco situaciones de tipo (b) y dos de estas pertenecían a una modalidad $(b)_{1}$, el valor correspondiente a dicha modalidad, en esa díada, era de 0.40 (2/5). Para analizar la significación de la distribución de cada modalidad se utilizó la prueba $t$ de student para una sola muestra.

3. Análisis de las modalidades de integración entre respuestas individuales y respuesta diádica, en función del grado de complejidad del ítem. Se calcularon proporciones de ocurrencia de manera similar al punto 2, pero al interior de cada uno de los cuatro grupos de ítems referidos a los niveles crecientes de complejidad, es decir, ítems de descripción de datos de la tabla, de comprensión de la estructura tabular, de inferencia particular de datos de la tabla y de inferencia global. Para comparar los cuatro niveles de complejidad de manera simultánea, se utilizó la prueba de Friedman; luego se efectuaron comparaciones por pares a través de la prueba de Wilcoxon (muestras relacionadas en ambos casos).

4. Análisis de las modalidades de integración entre respuestas individuales y respuesta diádica, en función de la composición sociocognitiva de la díada. Aquí también se calcularon valores de proporción, pero comparando díadas simétricas y asimétricas. Se utilizó la prueba $U$ de MannWhitney (muestras independientes).

\section{Resultados}

\section{Comparación de los desempeños (cantidad de ítems correctos) individual y diádico}

En primer lugar, este análisis se realizó al interior de las díadas simétricas, omitiendo las asimétricas, para poder comparar el desempeño individual y el diádico en condiciones equivalentes, lo cual no era posible si se incluían las díadas asimétricas, porque era esperable un efecto de "enseñanza" o de influencia preponderante por parte del sujeto más competente. Los resultados revelaron que el promedio de respuestas correctas bajo la condición diádica $(M=6.79, D E=1.91)$ fue mayor que el logrado en condición individual $(M=6.08$, $D E=1.38)$, con valores significativos $[t(47)=-2.66$, $p<0.050]$.

\section{Análisis de las modalidades de integración entre respuestas individuales y respuesta diádica de cada díada}

La tabla 4 presenta los valores descriptivos de cada modalidad de integración individuo-díada en la muestra general de díadas $(n=45)$.

Tabla 4

Descriptivos de las proporciones de las modalidades de integración individual-colectivo en la muestra general

\begin{tabular}{lcccccc}
\hline \multicolumn{1}{c}{ Respuestas individuales (Pretest) } & Respuesta colaborativa & $M$ & Mdn & $D E$ & Mín. & Máx. \\
\hline Ambas & $(\mathrm{a})_{1}$ & 0.93 & 1.00 & 0.13 & 0.50 & 1.00 \\
correctas (a) & $(\mathrm{a})_{2}$ & 0.07 & 0.00 & 0.13 & 0.00 & 0.50 \\
\hline Una correcta y & $(\mathrm{b})_{1}$ & 0.57 & 0.60 & 0.32 & 0.00 & 1.00 \\
una incorrecta (b) & $(\mathrm{b})_{2}$ & 0.27 & 0.20 & 0.28 & 0.00 & 1.00 \\
& $(\mathrm{~b})_{3}$ & 0.09 & 0.00 & 0.18 & 0.00 & 1.00 \\
\hline Ambas & $(\mathrm{c})_{1}$ & 0.10 & 0.00 & 0.18 & 0.00 & 0.66 \\
incorrectas (c) & $(\mathrm{c})_{2}$ & 0.32 & 0.33 & 0.31 & 0.00 & 1.00 \\
& $(\mathrm{c})_{3}$ & 0.11 & 0.00 & 0.20 & 0.00 & 1.00 \\
& $(\mathrm{c})_{4}$ & 0.13 & 0.00 & 0.23 & 0.00 & 1.00 \\
& $(\mathrm{c})_{5}$ & 0.23 & 0.20 & 0.26 & 0.00 & 1.00 \\
& $(\mathrm{c})_{6}$ & 0.08 & 0.00 & 0.15 & 0.00 & 0.50 \\
\hline
\end{tabular}

Nota. Cfr. las distintas modalidades de integración en tabla 3.

Fuente: elaboración propia. 
Los resultados revelaron que, cuando ambos sujetos mostraban respuestas iniciales individuales correctas (situación a), era muy probable que esta se trasladara a la respuesta diádica $\left((a)_{1}, t(44)=22.18\right.$, $p<0.001)$. Por contrapartida, la construcción diádica de una respuesta errónea $\left((\mathrm{a})_{2}\right)$ constituyó una situación excepcional. Por otro lado, cuando uno de los compañeros producía una respuesta inicial correcta y el otro no (situación b), en la mayoría de las ocasiones la respuesta diádica era idéntica a la respuesta correcta emitida en el pretest $((b), t(44)=5.02, p<0.001)$, y en menor medida se trataba de la respuesta incorrecta propuesta previamente $\left[(b)_{2}\right]$. De manera excepcional, ocurría que la respuesta diádica era incorrecta, sin que hubiera sido propuesta previamente por alguno de los compañeros $\left((b)_{3,} t(44)=-8.90, p<0.001\right)$.

Por último, cuando ambos sujetos mostraban respuestas incorrectas en el pretest (situación c), lo más probable es que la díada reprodujera la misma propuesta inicial, sobre todo si esta había sido emitida por ambos individuos en el pretest $\left((\mathrm{C})_{2}\right.$, $t(44)=3.27, p<0.010)$. También, aunque en menor medida, la díada podía construir una respuesta diádica correcta y nueva con respecto a los pretest individuales, especialmente cuando ambos sujetos habían propuesto la misma respuesta incorrecta en el pretest $\left((\mathrm{C})_{1}, t(44)=-2.67, p<0.050\right)$.

\section{Análisis de las modalidades de integración entre respuestas individuales y respuesta diádica, en función del nivel de complejidad de los ítems}

La tabla 5 presenta los valores descriptivos de cada modalidad, al interior de los cuatro tipos de ítems agrupados por nivel de complejidad en la interpretación de la tabla.

Tabla 5

Descriptivos de las proporciones de las modalidades de integración individual y colectivo, según la complejidad de los ítems

\begin{tabular}{|c|c|c|c|c|c|c|c|c|c|c|c|c|c|}
\hline \multirow{3}{*}{$\begin{array}{l}\text { Respuestas } \\
\text { individuales } \\
\text { (pretest) }\end{array}$} & \multirow{3}{*}{$\begin{array}{l}\text { Respuesta } \\
\text { colaborativa }\end{array}$} & \multicolumn{12}{|c|}{ Nivel de complejidad de los ítems } \\
\hline & & \multicolumn{3}{|c|}{ Descripción datos } & \multicolumn{3}{|c|}{$\begin{array}{l}\text { Comp. estruct. } \\
\text { tabular }\end{array}$} & \multicolumn{3}{|c|}{$\begin{array}{l}\text { Inferencia parcial } \\
\text { de datos }\end{array}$} & \multicolumn{3}{|c|}{$\begin{array}{l}\text { Inferencia } \\
\text { global }\end{array}$} \\
\hline & & $M$ & $M d n$ & $D E$ & $M$ & $M d n$ & $D E$ & $M$ & $M d n$ & $D E$ & $M$ & Mdn & $D E$ \\
\hline \multirow{5}{*}{$\begin{array}{l}\text { Ambas } \\
\text { correctas (a) } \\
\text { Una correcta } \\
\text { y una } \\
\text { incorrecta (b) }\end{array}$} & (a) & 0.99 & 1.00 & 0.06 & 0.69 & 1.00 & 0.45 & 0.16 & 0.00 & 0.37 & 0.09 & 0.00 & 0.26 \\
\hline & $(\mathrm{a})_{2}$ & 0.01 & 0.00 & 0.06 & 0.11 & 0.00 & 0.30 & 0.02 & 0.00 & 0.15 & 0.28 & 0.00 & 0.37 \\
\hline & (b) ${ }_{1}$ & 0.56 & 1.00 & 0.48 & 0.53 & 0.50 & 0.47 & 0.32 & 0.00 & 0.44 & 0.16 & 0.00 & 0.35 \\
\hline & $(b)_{2}$ & 0.03 & 0.00 & 0.13 & 0.20 & 0.00 & 0.36 & 0.30 & 0.00 & 0.44 & 0.18 & 0.00 & 0.37 \\
\hline & $(b)_{3}$ & 0.01 & 0.00 & 0.05 & 0.04 & 0.00 & 0.21 & 0.04 & 0.00 & 0.17 & 0.18 & 0.00 & 0.39 \\
\hline \multirow{6}{*}{$\begin{array}{l}\text { Ambas } \\
\text { incorrectas } \\
\text { (c) }\end{array}$} & (c) & 0.00 & 0.00 & 0.00 & 0.10 & 0.00 & 0.29 & 0.09 & 0.00 & 0.21 & 0.04 & 0.00 & 0.21 \\
\hline & (c) & 0.00 & 0.00 & 0.00 & 0.24 & 0.00 & 0.42 & 0.28 & 0.00 & 0.38 & 0.18 & 0.00 & 0.37 \\
\hline & $(\mathrm{c})_{3}$ & 0.02 & 0.00 & 0.15 & 0.00 & 0.00 & 0.00 & 0.05 & 0.00 & 0.18 & 0.14 & 0.00 & 0.31 \\
\hline & (c) & 0.00 & 0.00 & 0.00 & 0.00 & 0.00 & 0.00 & 0.14 & 0.00 & 0.31 & 0.10 & 0.00 & 0.27 \\
\hline & & 0.00 & 0.00 & 0.00 & 0.03 & 0.00 & 0.17 & 0.18 & 0.00 & 0.31 & 0.27 & 0.00 & 0.41 \\
\hline & & 0.04 & 0.00 & 0.21 & 0.00 & 0.00 & 0.00 & 0.04 & 0.00 & 0.13 & 0.11 & 0.00 & 0.30 \\
\hline
\end{tabular}

Nota. Cfr. las distintas modalidades de integración en tabla 3.

Fuente: elaboración propia.

En las situaciones en las que ambos sujetos de la díada mostraron respuestas correctas en el pretest (situación a), conforme se avanzaba en el nivel de dificultad, descendía significativamente la probabilidad de la díada de reproducir la respuesta correcta inicial $\left(x^{2}(3)=89.24, p<0.001\right)$.
Las diferencias mayores ocurrieron entre los dos niveles más simples, descripción y comprensión de la estructura tabular, y entre los dos niveles más complejos, inferencia particular y global. A su vez, en los niveles de dificultad superior se observó una mayor probabilidad de registrar una respuesta 
diádica incorrecta, a pesar de que ambos sujetos produjeron respuestas correctas individuales en el pretest $\left(x^{2}(3)=34.93, p<0.001\right)$.

En las díadas con una respuesta individual previa correcta y una individual incorrecta (situación b) se observó una disminución significativa $\left(x^{2}(3)=23.20\right.$, $p<0.001)$ ) de la repetición de la respuesta correcta $\left[\left(b_{1}\right)\right]$ en la condición diádica, a medida que aumentaba la complejidad de los ítems. Como en el paso analítico anterior, aquí también se observó un salto cualitativo entre los dos niveles inferiores, descripción y comprensión de tabla, por un lado, y los dos niveles superiores de inferencia, particular y global, por el otro. Respecto a los otros dos desenlaces posibles en la fase diádica, es decir, la construcción de una respuesta incorrecta similar $\left[(b)_{2}\right]$ o diferente $\left[(b)_{3}\right]$ a la respuesta incorrecta planteada por uno de los sujetos en el pretest, cabe observar cómo en los ítems más complejos la proporción de ocurrencia de ambas fue similar. En síntesis, en los ítems más simples se registró una tendencia muy parecida a la detectada sobre la muestra general, lo más probable es que la díada construyera una respuesta correcta similar a la propuesta por uno de los sujetos en el pretest. Sin embargo, en los ítems más complejos de inferencia, particular y global, las probabilidades de construir una respuesta diádica correcta o una incorrecta se equipararon. Incluso, se aumentó considerablemente la posibilidad de generar una respuesta diádica incorrecta inédita con respecto a las soluciones individuales previas.
Finalmente, el escenario donde ambos sujetos mostraron respuestas incorrectas (situación c), prácticamente no se registró en los ítems más simples de descripción de la tabla. En los ítems de segundo nivel, estructura tabular, emergió una tendencia parecida a la observada en la muestra general, salvo que se redujo la brecha entre la posibilidad de generar respuestas correctas, especialmente cuando ambas respuestas incorrectas del pretest eran iguales, y el mantenimiento de la misma respuesta incorrecta del pretest. En el tercer nivel fue donde más se observó la posibilidad de crear una respuesta nueva correcta a partir de dos previas incorrectas, similares o diferentes entre sí, aunque siempre fue mayor la probabilidad de repetir la respuesta incorrecta propuesta en el pretest. En el cuarto nivel, inferencia global, aumentó considerablemente la probabilidad de generar respuestas diádicas incorrectas diferentes a las incorrectas propuestas en el pretest $\left[(\mathrm{c})_{3}\right.$ y $\left.(\mathrm{c})_{6}\right]$.

Análisis de las modalidades de integración entre respuestas individuales y respuesta diádica, en función de la composición sociocognitiva de la díada (simétrica y asimétrica)

La tabla 6 muestra los valores descriptivos de cada modalidad de integración individuo-díada en las díadas simétricas y asimétricas.

Tabla 6

Descriptivos de las proporciones de las modalidades de integración individual-colectivo, según composición sociocognitiva de la díada

\begin{tabular}{|c|c|c|c|c|c|c|c|}
\hline \multirow{3}{*}{$\begin{array}{c}\text { Respuestas individuales } \\
\text { (Pretest) }\end{array}$} & \multirow{3}{*}{$\begin{array}{c}\text { Respuesta } \\
\text { colaborativa }\end{array}$} & \multicolumn{6}{|c|}{ Composición sociocognitiva } \\
\hline & & \multicolumn{3}{|c|}{ Simétrica } & \multicolumn{3}{|c|}{ Asimétrica } \\
\hline & & M & Mdn & $D E$ & M & Mdn & $D E$ \\
\hline \multirow{2}{*}{$\begin{array}{l}\text { Ambas } \\
\text { correctas (a) }\end{array}$} & $(\mathrm{a})_{1}$ & 0.91 & 1.00 & 0.16 & 0.96 & 1.00 & 0.09 \\
\hline & $(\mathrm{a})_{2}$ & 0.09 & 0.00 & 0.16 & 0.04 & 0.00 & 0.09 \\
\hline \multirow{3}{*}{$\begin{array}{l}\text { Una correcta y } \\
\text { una incorrecta }(b)\end{array}$} & (b) & 0.47 & 0.55 & 0.35 & 0.69 & 0.66 & 0.25 \\
\hline & $(b)_{2}$ & 0.30 & 0.25 & 0.32 & 0.24 & 0.17 & 0.23 \\
\hline & $(b)_{3}$ & 0.10 & 0.00 & 0.23 & 0.07 & 0.00 & 0.10 \\
\hline \multirow{6}{*}{$\begin{array}{l}\text { Ambas } \\
\text { incorrectas (c) }\end{array}$} & (c) ${ }_{1}$ & 0.13 & 0.00 & 0.19 & 0.06 & 0.00 & 0.16 \\
\hline & (c) ${ }_{2}$ & 0.38 & 0.42 & 0.29 & 0.25 & 0.00 & 0.32 \\
\hline & (c) ${ }_{3}$ & 0.11 & 0.00 & 0.13 & 0.11 & 0.00 & 0.26 \\
\hline & (c) ${ }_{4}$ & 0.08 & 0.00 & 0.14 & 0.18 & 0.00 & 0.29 \\
\hline & (c) ${ }_{5}$ & 0.24 & 0.20 & 0.22 & 0.23 & 0.00 & 0.31 \\
\hline & (c) & 0.06 & 0.00 & 0.11 & 0.11 & 0.00 & 0.19 \\
\hline
\end{tabular}

Nota. Cfr. las distintas modalidades de integración en tabla 3.

Fuente: elaboración propia. 
La principal diferencia entre díadas simétricas y asimétricas se refiere a la modalidad en la que un sujeto contaba con una respuesta correcta en el pretest, y el otro, con una respuesta incorrecta en la misma instancia, reiterándose la primera en la fase interactiva $\left(b_{1}\right)$. Esta modalidad predominó significativamente en las díadas asimétricas, como era de esperarse, por el liderazgo cognitivo del sujeto más competente.

\section{Discusión}

El objetivo general del estudio fue analizar la integración entre el producto cognitivo individual y el producto cognitivo diádico, en una tarea de comprensión de una tabla de frecuencias. El foco de interés estuvo puesto en la contraposición entre comprensión individual, previa a la interacción, y la posterior coconstrucción de una comprensión diádica, a partir de la interacción. Por tanto, se privilegió el estudio del producto cognitivo, puntualmente su calidad y cualidad, logrado bajo ambas condiciones sociales: individual y diádica. De allí derivaron cuatro objetivos específicos, los cuales dieron lugar a una serie de conclusiones que se describen a continuación.

El primer objetivo específico proponía comparar los desempeños generales (cantidad de ítems correctos) individual y diádico, con la finalidad de corroborar la hipótesis clásica de la superioridad de la segunda sobre la primera (Gilly, 1992; Peralta y Castellaro, 2018; Peralta et al., 2012; Roselli, 2016). Efectivamente, los resultados aquí observados son coherentes con el presupuesto básico del socioconstructivismo, es decir, la calidad de la comprensión de la tabla es, en general, mayor en la condición diádica que en la individual.

El segundo objetivo específico fue analizar modalidades de integración entre la comprensión individual de la tabla (respuesta previa a la interacción) y la posterior coconstrucción de una comprensión diádica, a partir de la interacción. El punto de partida fue la identificación de tres situaciones iniciales en el pretest: (a) ambos sujetos con respuestas individuales correctas, (b) un sujeto con una respuesta correcta y el otro con una incorrecta, y (c) ambos sujetos con respuestas incorrectas. Al interior de cada situación, se analizaron posibles desenlaces en función de la respuesta diádica construida posteriormente: primero, cuando ambos compañeros poseen respuestas correctas, y por tanto, idénticas, en el pretest, esta tiende a persistir en la fase diádica, con excepción de los ítems más dificultosos, tal como se observó al analizar esta variable mediadora, lo cual se discute más abajo. Segundo, cuando ambos compañeros tienen respuestas incorrectas y similares en el pretest, también se observa una tendencia similar a lo anterior, aunque en menor grado, puesto que surge la posibilidad de construcción diádica de una respuesta inédita, que puede ser correcta, en el mejor de los casos, o incorrecta. Por último, hay que efectuar una distinción entre aquellas situaciones en las que ambos sujetos cuentan con perspectivas iniciales diferentes entre sí. Por un lado, cuando uno ha construido una solución correcta y el otro no, lo más esperable es que predomine la solución del primero o, en menor medida, la solución del segundo. Pero, es poco esperable que la díada construya una solución inédita o diferente a una de las dos soluciones aportadas inicialmente. Por otro lado, cuando los sujetos presentan respuestas individuales incorrectas y diferentes entre sí, lo más esperable es que la díada construya: (a) una respuesta similar a la propuesta por uno de los sujetos, o (b) una respuesta inédita en comparación con cualquiera de las propuestas previamente. Cuando ocurre esto último, lo más probable es que la solución construida por la díada sea correcta.

Podrían establecerse analogías conceptuales entre ciertas modalidades observadas y paradigmas teóricos generales. Por ejemplo, cuando ambos sujetos producen respuestas incorrectas y diferentes entre sí en el pretest, esto podría entenderse como una situación potencial de conflicto sociocognitivo (Peralta y Roselli, 2016). Los resultados sugieren que, en este caso, la mayor parte de las díadas no construyeron una respuesta inédita con respecto al pretest individual, sino que tendían a reproducir una de las soluciones propuestas de antemano. A pesar de ello, en aquellas ocasiones menos frecuentes en las que sí lograron construir una solución diádica inédita, la mayor parte de las veces esta fue correcta.

Otra situación interesante se produjo cuando en el pretest individual ambos sujetos emitieron respuestas diferentes entre sí y la díada finalmente adoptó una de estas posiciones. Este hallazgo permitió observar una clara situación de influencia social, positiva o negativa, dependiendo de si la díada logra construir una respuesta final acertada o desacertada. Tal como se ha reportado en otros 
trabajos (Castellaro y Roselli, 2018b; Quiamzade, Mugny y Butera, 2014; Quiamzade, Mugny y Darnon, 2009), estos procesos de influencia social pueden estar regulados por diferentes factores; en algunos casos, pueden depender principalmente de un elemento cognitivo, igualdad o diferencia entre los niveles de competencia específica de los compañeros. Sin embargo, en otras situaciones, puede estar condicionado prioritariamente por elementos psicosociales, por ejemplo, rasgos de personalidad, género, afinidad socioafectiva, entre otros.

El tercer objetivo específico proponía incluir la complejidad de los ítems como variable interviniente. Así, se compararon cuatro niveles en la comprensión de la tabla (Gabucio et al., 2010): (a) describir sus datos; (b) comprender su estructura tabular; (c) inferir datos puntuales, inferencia de primer nivel; y (d) realizar inferencias globales, inferencia de segundo nivel. Los hallazgos revelaron una influencia significativa de esta variable sobre la modalidad de integración de la producción individual-colectiva, tal como se ha observado en trabajos previos, aunque estos estén referidos a otro tipo de tareas (Arterberry et al., 2007; Liskala et al., 2011; Ogden, 2000). En lo que respecta a la distinción original de los cuatro niveles de complejidad, puede concluirse que el salto cualitativo más notorio se produjo entre los dos niveles básicos -describir datos y comprender la estructura tabular- y los dos superiores -inferencia particular y global-, pues las variaciones más marcadas se observaron en ese punto. En ese sentido, podría plantearse que la distinción conceptual más importante se da entre describir aspectos directamente observables de la tabla e inferir conclusiones e ideas que trascienden los aspectos directamente observables de la tabla. Por ejemplo, en los niveles de dificultad superior, aún en los casos en que ambos sujetos mostraron respuestas correctas en el pretest, se observó una mayor probabilidad de registrar una respuesta colaborativa incorrecta. Esto muestra que no siempre y necesariamente la producción colectiva conduce a un progreso cognitivo, pues cuando la dificultad cognitiva es muy alta, la respuesta individual, aunque sea correcta, no es firme y está sujeta a dudas y cambios regresivos. Se podría decir que, ante una respuesta correcta no consolidada, la discusión interactiva puede generar un cierto desconcierto o vacilación.
El cuarto objetivo específico fue analizar la integración entre comprensión individual y diádica en función de la composición sociocognitiva de la díada (simétrica y asimétrica), lo cual arrojó diferencias significativas en relación con la modalidad en la que, partiendo de una situación inicial de diferencia cognitiva, una respuesta correcta y otra incorrecta, predominaba la respuesta correcta en la fase diádica. Esta situación es entendible en términos de la influencia social ejercida por el sujeto más competente, lo que coincide con trabajos previos que postularon un efecto diferenciador por parte de la composición sociocognitiva (Asterhan et al., 2014; Buchs y Butera, 2009; Castellaro y Roselli 2018a, b; Schmitz y Winskel, 2008).

Al mismo tiempo, hay que considerar que el presente trabajo tiene un carácter complementario respecto a dichos antecedentes, ya que estos estuvieron centrados en el análisis de la interacción sociocognitiva, y este se concentró en la calidad y la cualidad de la respuesta final o resultado cognitivo. Por lo tanto, pone en evidencia la distinción teórica entre producto sociocognitivo y proceso sociocognitivo.

En relación con este último punto, y a modo de cierre, se plantea continuar el estudio a partir del análisis del proceso interactivo, que media entre la comprensión individual (pretest) y la comprensión final lograda por la díada a partir de la interacción. Si bien este trabajo se concentró en comparar ambos resultados finales, se entiende que la indagación del proceso interactivo intermedio aportaría nuevos elementos para una mayor comprensión del fenómeno. Básicamente, el análisis del proceso de construcción diádica permitiría diferenciar entre una interacción de negociación de posiciones individuales y otra de construcción colaborativa auténtica. Esto implicaría entrar en el análisis del discurso conversacional de los sujetos. Este análisis procesual posibilitaría, además, discriminar entre el aspecto puramente cognitivo de la interacción y el aspecto socioafectivo, permitiendo una mejor comprensión de la variable simetría-asimetría cognitiva de los sujetos, al incorporar factores extracognitivos de influencia social, tales como rasgos de dominancia o ascendencia personal. Otro aspecto a tener en cuenta para futuras investigaciones es la ampliación de la investigación a otros sistemas externos de representación, tales como los mapas conceptuales y demás diagramas de representación cognitiva. 
En cuanto a la posible aplicación de los resultados, sin duda, el campo educativo, en especial el de la didáctica, es el principal beneficiario. Las conclusiones obtenidas por este y otros estudios que puedan sucederse aportarían al permanente debate entre los educadores con posiciones individualistas y socioconstructivistas, lo cual permitiría la implementación de didácticas que integren ambas perspectivas.

\section{Referencias}

Arterberry, M., Cain, K., \& Chopko, S. (2007). Collaborative Problem Solving in Five-Year-Old Children: Evidence of Social Facilitation and Social Loafing. Educational Psychology, 27(5), 577-596. doi: 10.1080/01443410701308755

Asterhan, C., Schwarz, B., \& Cohen-Eliyahu, N. (2014). Outcome Feedback During Collaborative Learning: Contingencies Between Feedback and Dyad Composition. Learning and Instruction, 34, 1-10. doi:10.1016/j. learninstruc.2014.07.003

Buchs, C., \& Butera, F. (2009). Is a Partner's Competence Threatening During Dyadic Cooperative Work? It Depends on Resource Interdependence. European Journal of Psychology of Education, 24(2), 145-154. doi: 10.1007/BF03173007

Castellaro, M. (2017). La interacción social como clave del desarrollo cognitivo: Aportes del socioconstructivismo a la Psicología. Revista de Psicología Digital, 5, 1-14. Recuperado de http://psicologiadigital.unr.edu.ar/?p=942

Castellaro, M., \& Roselli, N. (2015). Peer Collaboration in Children According to Age, Socioeconomic Context and Task. European Journal of Psychology of Education, 30, 63-80. doi: 10.1007/s10212-014-0228-3

Castellaro, M. y Roselli, N. (2018a). Interacción sociocognitiva entre pares en situaciones simétricas y asimétricas de competencia epistémica. Revista de Psicología, 36(1), 333365. doi: 10.18800/psico.201801.011

Castellaro, M. y Roselli, N. (2018b). Resolución colaborativa de problemas lógicos en condiciones de simetría y asimetría cognitiva.
Revista Propósitos y Representaciones, 6(1), 135-166. doi: 10.20511/pyr2018.v6n1.196

Gabucio, F., Martí, E., Enfedaque, J., Gilabert, S. y Konstantinidou, A. (2010). Niveles de comprensióndelastablasenalumnos deprimaria y secundaria. Infancia y Aprendizaje, 22(2), 183-197. doi: 10.1174/113564010791304528

García-Mila, M., Martí, E., Gilabert, S., \& Castells, M. (2014). Fifth Through Eighth Grade Students' difficulties in Constructing Bar Graphs: Data Organization, Data Aggregation, and Integration of a Second Variable. Mathematical Thinking and Learning, 16, 201-233. doi: 10.1080/10986065.2014.921132

Gilly, M. (1992). Introducción: Interacciones entre pares y construcciones cognitivas: modelos explicativos. En A.N. Perret-Clermont y M. Nicolet (Comps.), Interactuar y Conocer. Desafíos y regulaciones sociales en el desarrollo cognitivo (pp. 23-31). Buenos Aires: Miño y Dávila.

Liskala, T., Vauras, M., Lehtinen, E., \& Salonen, P. (2011). Socially Shared Metacognition of Dyads of Pupils in Collaborative Mathematical Problem-Solving Processes. Learning and Instruction, 21, 379-393. doi: 10.1016/j. learninstruc.2010.05.002

Maita, M., Mareovich F., \& Peralta, O. (2014). Intentional Teaching Facilitates Young Children's Comprehension and Use of a Symbolic Object. Journal of Genetic Psychology, 175(5), 401415. doi: 10.1080/00221325.2014.941320

Martí, E., García-Mila, M., Gabucio, F., \& Konstantinidou, K. (2011). The Construction of a Double-Entry Table: a Study of Primary and Secondary School's Students Difficulties. European Journal of Psychology of Education, 26(2), 215-234. doi: 10.1007/s10212-0100046-1

Martí, E., Scheuer, N., Cavalcante, S., Trench, M., \& Brizuela, B. M. (2016). Symbolic Representation of the Number Three: a Study with Three-YearOld Children from Contrasting Socioeconomic Environments. Journal of Cognitive Psychology, 28(6), 743-755. doi:10.1080/20445911.2016. 1188821

Mejía-Arauz, R., Rogoff, B., Dayton, A., \& HenneOchoa, R. (2018). Collaboration or Negotiation: 
Two Ways of Interacting Suggest How Shared Thinking Develops. Current Opinion in Psychology, 23, 117-123. doi: 10.1016/j. copsyc.2018.02.017

Ogden, L. (2000). Collaborative tasks, collaborative children: An analysis of reciprocity during peer interaction at Key Stage 1. British Educational Research Journal, 26(2), 211-226. doi:10.1080/01411920050000953.

Peralta, N. y Castellaro, M. (2018). Interacción e intersubjetividad: Investigando sus beneficios en el ámbito educativo. En J. Faccendini, P. Martino, M. Sironi y M. Terrádez (Comps.), Caleidoscopio. Prácticas y clínicas Psi en la Universidad (pp. 247-259). Rosario: UNR.

Peralta, N. y Roselli, N. (2016). Conflicto sociocognitivo e intersubjetividad: Análisis de las interacciones verbales en situaciones de aprendizaje colaborativo. Psicología, Conocimiento y Sociedad, 6(1), 90-113. Recuperado de https://revista.psico.edu.uy/ index.php/revpsicologia/article/view/313

Peralta, N., Roselli, N. y Borgobello, A. (2012). El conflicto sociocognitivo como instrumento de aprendizaje en contextos colaborativos. Revista Interdisciplinaria, 29(2), 325-338. Recuperado de http://www.scielo.org.ar/pdf/interd/v29n2/ v29n2a08.pdf

Peralta, O. y Salsa, A. (2011). Instrucción y desarrollo en la comprensión temprana de fotografías como objetos simbólicos. Anales de Psicología, 27(1), 118-125. Recuperado de https://revistas. um.es/analesps/article/view/113541

Peralta, O., Salsa, A., Maita, M. R., \& Mareovich, F. (2013). Scaffolding Young Children's Understanding of Symbolic Objects. Early Years: An International Journal of Research and Development, 33(3), 266-274. doi: 10.1080/09575146.2012.732042

Pérez, M., Martí, E. y Pozo, J. (2010). Los sistemas externos de representación como herramientas de la mente. Infancia y Aprendizaje, 22(2), 133-147. doi: 10.1174/113564010791304519

Pérez-Echeverría, M. P., Postigo, Y. y Marín, C. (2010). Las habilidades gráficas de los estudiantes universitarios: ¿cómo comprenden las gráficas los estudiantes de psicología?
Cultura y Educación, 22(2), 215-229. doi: 10.1174/113564010791304537

Psaltis, C., \& Zapiti, C. (2014). Interaction, Communication and Development: Psychological Development as a Social process. United Kingdom: Routledge. Recuperado de https://www.routledge.com/InteractionCommunication-and-DevelopmentPsychological-development-as/Psaltis-Zapiti/p/ book/9780415643870

Psaltis, C., Duveen, G., \& Perret-Clermont, A. (2009). The Social and the Psychological: Structure and Context in Intellectual Development. Human Development, 52(5), 291-312. doi: 10.1159/000233261

Quiamzade, A., Mugny, G., \& Butera, F. (2014). Psychologie Sociale de la Connaissance. Grenoble: Presses Universitaires de Grenoble.

Quiamzade, A., Mugny, G., \& Darnon, C. (2009). The Coordination of Problem Solving Strategies: When Low Competence Sources Exert More Influence on Task Processing than High Competence Sources. British Journal of Social Psychology, 48(1), 159-182. doi: 10.1348/014466608X311721

Rodríguez, J., Martí, E., \& Salsa, A. (2018). Symbolic Representations and Cardinal Knowledge in 3- and 4-Year-Old Children. Cognitive Development, 48, 235-243. doi: 10.1016/j.cogdev.2018.09.004

Roselli, N. (2016). Modalities to Collaborate in the Social Construction of Conceptual Maps: A Comparison Between Individual and Collective Productions. American Journal of Educational Research, 5(10), 1058-1064. doi: 10.12691/ education-5-10-7

Salsa, A. y Gariboldi, M. B. (2017). Experiencia con símbolos y comprensión de dibujos en niños pequeños de distintos contextos socioeconómicos. Avances en Psicología Latinoamericana, 36(1), 29-43. doi: 10.12804/ revistas.urosario.edu.co/apl/a.4332

Salsa, A. y Peralta, O. (2009). La lectura de material ilustrado: Resultados de una intervención con madres y niños pequeños de nivel socioeconómico bajo. Infancia y Aprendizaje, 32(1), 3-16. doi: 10.1174/021037009787138202 
Salsa, A., \& Vivaldi, R. (2017). Developmental Changes in Early Comprehension and Production of Drawings: Evidence from Two Socioeconomic Backgrounds. The Journal of Genetic Psychology, 178(4), 217-228. doi: 10.1080/00221325.2017.1328385
Schmitz, M., \& Winskel, H. (2008). Towards Effective Partnerships in a Collaborative Problem-Solving Task. British Journal of Educational Psychology, 78(4), 581-596. doi: 10.1348/000709908X281619

Para citar este artículo / To cite this article / Para citar este artigo: Castellaro, M. y Roselli, N. (2020). Comprensión individual y diádica de tablas de frecuencias en alumnos de escolaridad primaria. Pensamiento Psicológico, 18(1), 57-70. doi:10.11144/Javerianacali. PPSI18-1.cidt 